\title{
Synergistic Effects of Taurine and Temozolomide Via Cell Proliferation Inhibition and Apoptotic Induction on U-251 MG Human Glioblastoma Cells
}

\author{
Thanakorn Surarak $^{1 *}$, Pathanin Chantree ${ }^{2,3}$, Kant Sangpairoj ${ }^{2,3}$
}

\begin{abstract}
Objective: The combination treatment is a way to improve the therapeutic strategy of temozolomide (TMZ) -resistant glioblastoma (GBM). Taurine (TAU) has the potential to inhibit growth in various cancer cells. The aim of this study was to examine the combined effects of TMZ and TAU on cultured human GBM, U-251 MG cells. Methods: The cells were incubated with TMZ, TAU, and the combination of both in various ratios. MTT assay was performed to measure the cell viability of the treatments and then the synergistic interactions were evaluated by the Chou-Talalay method. The cell cycle and apoptotic properties of the combined treatment on U-251 MG cells were examined by flow cytometry. The Hoechst 33342 stainings were applied to visualize the morphologic change in the apoptotic process. Results: The combined treatment with a dose reduction of each expressed synergistic effect on the decrease of cell viability. The study on the cell cycle resulted in G2/M phase arrest with increasing apoptotic cells in the SubG1 phase. Moreover, the apoptotic effects of the combinations on U-251 MG cells were explained by the increase of apoptotic cells in both early and late stages and illustrated by some characteristics of the apoptotic process including condensed chromatin and fragmented nuclei. Conclusion: The study showed that the combination between TMZ and TAU has a potential in anticancer properties against $\mathrm{U}-251 \mathrm{MG}$ manifested by the induction of $\mathrm{G}_{2} / \mathrm{M}$ arrest and apoptosis. These results suggest that this combination may be useful to enhance the efficacy and reduce some adverse events of GBM treatment in the future.
\end{abstract}

Keywords: Temozolomide- taurine- U-251 MG cells- synergism- glioblastoma

Asian Pac J Cancer Prev, 22 (12), 4001-4009

\section{Introduction}

Glioblastoma or glioblastoma multiforme (GBM) is a brain tumor derived from an abnormal astrocyte. It is the most common brain tumor and has been found in people worldwide, including in Thailand (Miranda-Filho et al., 2017; Witthayanuwat et al., 2018). Major signs and symptoms of GBM include motor weakness in the beginning, progressive headache, and convulsion. Other signs and symptoms depend on brain areas that the tumor invades into such as hemiparesis, visual loss, and aphasia. The definite cause of this tumor is unknown; however, there are some theories proposed that it might cause by genetic factors, race or, people who have ever received radiation therapy on head-to-neck area (Urbańska et al., 2014). This disease has a poor prognosis, and its survival rate is approximately 13-16 months (Shergalis et al., 2018; Urbańska et al., 2014; Bleeker et al., 2012). The current standard treatments for GBM patients include surgical resection, radiation therapy, and chemotherapy. At present, near-total surgical resection of this tumor has been performed along with radiation therapy and chemotherapy for the prevention of recurrence. However, glioblastoma has a higher recurrent rate and has more severity as well as drug resistance after recurrence (Urbańska et al., 2014).

Temozolomide (TMZ) is a prodrug that is rapidly absorbed and hydrolyzed to be an active form, monomethyl triazene 5-(3-methyltriazen-1-yl)-imidazole4-carboxamide (MTIC). Because of lipophilicity with a small molecule, this agent easily penetrates through bloodbrain barrier into the brain. Furthermore, TMZ belongs to DNA alkylation group because the active form, MTIC plays a role to add the methyl group at the nitrogenous bases on DNA resulting in $\mathrm{G}_{2} / \mathrm{M}$ arrest and then apoptosis. TMZ is the first-line drug of chemotherapy together with radiation therapy for the treatment of glioblastoma. However, the combination between TMZ and radiation therapy cannot completely cure the disease, moreover; the 
patients also have a chance to experience recurrence of the disease (Lee et al., 2016). The common adverse effects of TMZ are myelosuppression, especially neutropenia and thrombocytopenia (Zhang et al., 2012).

Taurine (TAU) is a sulfur-containing amino acid that can be synthesized from methionine or cysteine mainly in the liver. TAU has been found mostly in neurons in the central nervous system and skeletal muscle cells (Seidel et al., 2018). Other sources of TAU have also been found in natural food such as meat, fish, and seafood as well as in energy drinks as a popular ingredient (Curran et al., 2017). It plays a role in the physiological processes in the body such as the development of the fetus, lung function, mitochondrial function, anti-oxidative defense, $\mathrm{Ca}^{2+}$ homeostasis (Goron et al., 2018; Schaffer et al., 2018). Previously, TAU has also exerted some pharmacological studies have been found that it has the potential to inhibit the proliferation and induce apoptosis in several types of cancer cells including human colon cancer cells (Zhang et al., 2014), human nasopharyngeal carcinoma cells(He et al., 2018), colorectal cancer cells (Liu et al., 2018), breast cancer cells (Zhang et al., 2015), human lung cancer cells (Tu et al., 2018) and glioblastoma cells (Gao et al., 2016) both in In vitro and xenograft model.

Following the principle of chemotherapy, a physician usually prescribes a combination of the medications to treat the varieties of cancer. Previous studies have reported that the combination of TMZ and resveratrol enhanced cytotoxicity of TMZ in U87 MG (Lin et al., 2012) and using of TMZ together with chloroquine reduced the growth of GBM by $\mathrm{G}_{2} / \mathrm{M}$ arrest (Lee, et al., 2015) as well as the combined TMZ with caffeine increased cytotoxicity and reduced colony formation of GMB ( $\mathrm{Li}$ et al., 2018). Moreover, the study in the combination of TAU and cyclophosphamide has been reported that it reduced Ehrlich ascites carcinoma proliferation in in vivo (Ibrahima et al., 2018). Additionally, the combined medications provide some benefits such as enhancing the efficacy of treatment and reduction of adverse effects of the medications.

Interestingly, TMZ combined with TAU has no study in glioblastoma. This study aims to investigate the antitumor effect of the combination between TMZ and TAU on glioblastoma. The results might pave the way to develop a new treatment strategy for glioblastoma in the future.

\section{Materials and Methods}

\section{Chemical and cell culture}

Temozolomide (TMZ) and taurine (TAU) were obtained from Sigma-Aldrich, St. Louis, MO. (Product Number: T2577 and T4571). The $100 \mathrm{mM}$ of TMZ stock solution and $400 \mathrm{mM}$ of TAU stock solution were prepared by dissolving in dimethyl sulfoxide (DMSO) and distilled water, respectively and then they were stored at $-20^{\circ} \mathrm{C}$ until use. U-251 MG cell line was purchased from Cell Line Services, Germany. Cells were cultured in DMEM medium supplemented with $1 \%$ penicillin-streptomycin $(10,000 \mathrm{U} / \mathrm{mL})$ and $10 \%$ heat-inactivated fetal bovine serum (Gibco BRL, Gaithersburg, Md., U.S.A.) and maintained at $37^{\circ} \mathrm{C}$ in an incubator containing $5 \% \mathrm{CO}_{2}$ and $95 \%$ humidity.

\section{Cytotoxicity analysis and synergistic interaction evaluation}

To determine the half-maximal inhibitory concentration $\left(\mathrm{IC}_{50}\right)$ of TMZ and TAU, U251-MG cells were seeded into a 96-well plate overnight and then treated with diluted TMZ or TAU in various concentrations for $24 \mathrm{~h}$ and 48 h. Afterward, the MTT working solution (Sigma-Aldrich, USA) was added to each well at a final concentration of $30 \mu \mathrm{g} / \mathrm{mL}$ and further incubated for $3 \mathrm{~h}$. OD values were measured by using a spectrophotometer with wavelengths at 562 and $630 \mathrm{~nm}$ and the results were used to calculate $\mathrm{IC}_{50}$ value. Thereafter, U-251 MG cells were treated with nine concentrations of combined doses between TMZ and TAU including $130 \%, 100 \%$ and $70 \%$ of $\mathrm{IC}_{50}$ of TAU, and $100 \%, 75 \%$, and $50 \%$ of maximal concentration of dissolved TMZ in a $3 \times 3$ matrix table for $24 \mathrm{~h}$ and $48 \mathrm{~h}$. The treated cells were analyzed by MTT assay. The synergistic effects were further evaluated by CompuSyn software (ComboSyn, Inc.) based on the theorem of Chou-Talalay in a previous study (Chou, 2010).

\section{Analysis of apoptotic cells by flow cytometry}

Cells were treated with a combination between TMZ and TAU or a single substance for $24 \mathrm{~h}$ and $48 \mathrm{~h}$. After that, treated cells were analyzed for the distribution of apoptotic stages by using Alexa Fluor ${ }^{\mathrm{TM}} 488$ Antibody Labeling Kit (Invitrogen ${ }^{\mathrm{TM}}$ ). Briefly, $1 \times 10^{5}$ harvested cells were incubated with Alexa Fluor ${ }^{\mathrm{TM}} 488$ Annexin $\mathrm{V}$ and Propidium iodide (PI) in the binding buffer and incubated for $15 \mathrm{~min}$ at room temperature in the dark. The distribution of apoptotic stages was evaluated by the FACSCalibur flow cytometer (Becton Dickinson, USA).

Analysis of apoptosis by determining nuclear condensation

The nuclear condensation and fragmentation were determined by Hoechst 33342 staining (Sigma-Aldrich, USA). After treatment with a combination between TMZ and TAU or a single substance, the culture media were discarded, and cells were washed in PBS. Afterward, treated cells were incubated with $10 \mathrm{mg} / \mathrm{mL}$ Hoechst stock solution diluted at 1.5:1,000 in PBS for 10 minutes in a dark condition at room temperature. Lastly, cells were washed 3 times in PBS and visualized by a fluorescence microscope at $480 \mathrm{~nm}$.

\section{Flow cytometry analysis of DNA content}

Treated cells were evaluated cell cycle distribution by using PI staining. Briefly, after incubated with various concentrations of the combination doses between TMZ and TAU for $24 \mathrm{~h}$ and $48 \mathrm{~h}, 2 \times 10^{5}$ treated cells were fixed with $81 \%$ ethanol and overnight incubated in a $-20^{\circ} \mathrm{C}$. Cells were washed twice in PBS to remove the ethanol. Cells were then re-suspended in FxCycle ${ }^{\mathrm{TM}} \mathrm{PI} / \mathrm{RNase}$ Staining Solution (InvitrogenTM) and incubated in the dark at room temperature for $15 \mathrm{~min}$. The DNA content was analyzed using the FACSCalibur flow cytometer (Becton Dickinson, USA). 


\section{Immunoblotting}

After treatment with a combination between TMZ and TAU or a single substance, total cellular proteins were obtained using RIPA cell lysis buffer (Cell Signaling Technology, USA) and determined the concentrations by using Bicinchoninic acid (BCA) (Thermo Scientific, USA). From each sample, $10 \mu \mathrm{g}$ of total proteins were separated by using $12.5 \%$ SDS-PAGE and further transferred onto PVDF membranes. The membranes were blocked in blocking buffer (5\% BSA in Tris-buffered saline (TBS)) for $1 \mathrm{~h}$ then incubated with primary antibodies (Cell Signaling Technology, USA) including rabbit anti-Bax, rabbit anti-Bcl-2, rabbit anti-AKT, rabbit anti-pAKT, and rabbit anti- $\beta$-actin (Santa Cruz Biotechnology, USA) diluted in $1 \%$ BSA in TBS at $4{ }^{\circ} \mathrm{C}$ overnight. The membranes were washed in TBS with $0.1 \%$ tween (TBST) and further incubated with horseradish peroxidase (HRP) conjugated secondary antibody (Abcam, USA) diluted in TBS at 1:20,000 for $1 \mathrm{~h}$ at room temperature. The corresponding targeted proteins were visualized by interaction with $\mathrm{TMB}$ substrate (InvitrogenTM, USA) and using colorimetric detection. The intensity of protein bands was quantified by using ImageJ software.

\section{Statistical analysis}

Data were expressed as the mean \pm SEM obtained from triplicate experiments. Statistical analysis was performed using one-way analysis of variance (ANOVA) with Turkey post hoc test provided in GraphPad Prism 6 software. The values obtained in the assays were statistically significant when $\mathrm{P}<0.05$.

\section{Results}

Cytotoxicity effect of Taurine, temozolomide and the combinations on U-251 MG cells

After treatment for $24 \mathrm{~h}$ and $48 \mathrm{~h}$ with various concentrations of TAU or TMZ on U-251 MG cells, the MTT assay was performed to investigate the cytotoxicity of each of the substances. The percentages of cell viability of U-251 MG cells of the TAU treatment were decreased in a concentration-dependent manner from 5 to $50 \mathrm{mM}$ compared with the control group (Figure 1a). The 50\% inhibitory concentrations $\left(\mathrm{IC}_{50}\right.$ ) of TAU for U-251 MG cells were $20.23 \pm 1.99 \mathrm{mM}$ and $19.72 \pm 2.53 \mathrm{mM}$ at 24 $\mathrm{h}$ and $48 \mathrm{~h}$, respectively. At $500 \mu \mathrm{M}$ of temozolomide, $\%$ cell viability at $24 \mathrm{~h}$ and $48 \mathrm{~h}$ were $93.92 \pm 3.43$ and $97.83 \pm 7.83$, respectively (Figure $1 \mathrm{~b}$ ). The combinations were determined as the combination codes (Table 1). The cell viability of the combinations was investigated by MTT assay. The percentages of the cell viability of the combination A1, A2, A3, B1, B2, B3 were significantly decreased when compared with control and were comparable with TAU24 at $24 \mathrm{~h}$ and $48 \mathrm{~h}$. Similarly, at only $48 \mathrm{~h} \%$ cell viability of the combinations $\mathrm{C} 1$ and $\mathrm{C} 2$ was comparable with TAU24 (Figure 1c).

The percentage of cell death of the combinations was analyzed using CompuSyn software based on Chou-Talalay method to evaluate the interactions indicated by the combination indices $(\mathrm{CI})$ where $\mathrm{CI}<1$, = 1 , and $>1$ represent synergistic, additive, and antagonistic interaction, respectively. As result shown in Figure 1D, all the combinations obtained $\mathrm{CI}<1$. Moreover, the more CI value was decreased, the stronger synergistic interaction was indicated. Thus, C2 obtained the most synergistic interaction among other combinations.

Analysis of apoptotic cells of the combinations by flow cytometry in U-251 MG cells

Apoptotic cells illustrated a specific situation of the cell membrane to flip the phosphatidylserine molecule on the surface of the cell membrane from inside to outside. Because of the alteration of the cell membrane, some molecules of dyes can easily penetrate the cell. Thus, apoptotic cells are illustrated by staining with Annexin V and PI. In this study, apoptotic events were analyzed by flow cytometry. The result suggested that many Annexin $\mathrm{V}$ and PI-positive cells in treated cells with B2 and C2 were increased when compared with a control group at $24 \mathrm{~h}$ (Figure 2a, d, e) and $48 \mathrm{~h}$ (Figure 2f, i, j). Moreover, the percentages of treated cells distributed in early and late apoptotic phases were significantly increased after treatment with $\mathrm{C} 2$ at $48 \mathrm{~h}$ (Figure $2 \mathrm{k}, 1$ ). The present study confirmed that $\mathrm{C} 2$, which was the combination between $12 \mathrm{mM}$ of TAU and $375 \mu \mathrm{M}$ of TMZ (ratio TAU:TMZ = 32:1), could induce apoptosis in U-251 MG cells with a synergistic effect compared to a higher concentration of

Table 1. The Combination Codes Indicate Concentrations of TAU and TMZ and CI at $24 \mathrm{~h}$ and $48 \mathrm{~h}$

\begin{tabular}{lcccccc}
\hline Combination codes & TAU $(\mathrm{mM})$ & TMZ $(\mu \mathrm{M})$ & \multicolumn{2}{c}{$24 \mathrm{~h}$} & \multicolumn{2}{c}{$48 \mathrm{~h}$} \\
& & & Effect & CI & Effect & CI \\
\hline A1 & 24 & 500 & 0.545 & 0.93120 & 0.600 & 0.92537 \\
A2 & 24 & 375 & 0.564 & 0.87512 & 0.617 & 0.84678 \\
A3 & 24 & 250 & 0.554 & 0.89105 & 0.581 & 0.92805 \\
B1 & 18 & 500 & 0.498 & 0.80599 & 0.545 & 0.86359 \\
B2 & 18 & 375 & 0.495 & 0.80152 & 0.518 & 0.90885 \\
B3 & 18 & 250 & 0.468 & 0.85082 & 0.512 & 0.89337 \\
C1 & 12 & 500 & 0.350 & 0.85169 & 0.460 & 0.80583 \\
C2 & 12 & 375 & 0.363 & 0.79739 & 0.493 & 0.69177 \\
C3 & 12 & 250 & 0.347 & 0.81543 & 0.414 & 0.85214 \\
\hline
\end{tabular}

$\mathrm{CI}$, combination index; TAU, taurine; TMZ, temozolomide 
(a)

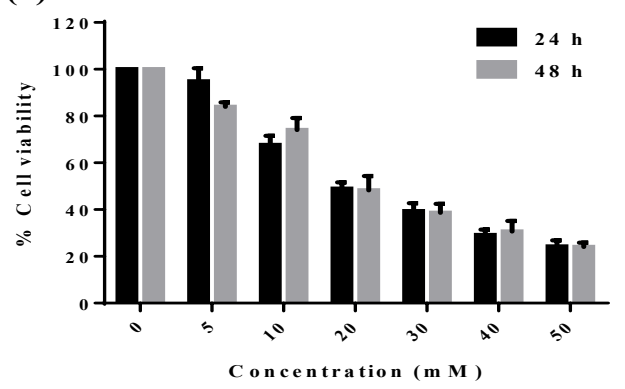

(b)

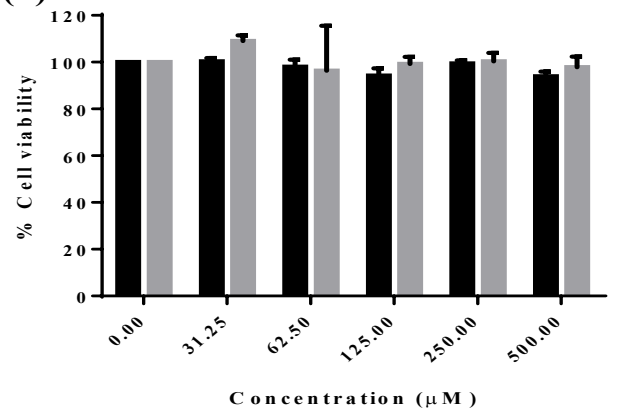

(c)

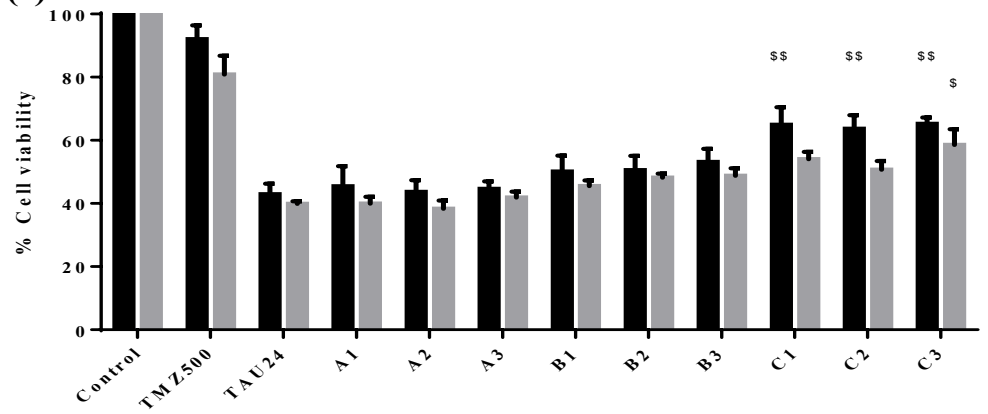

Figure 1. Percent of Cell Viability of U-251 MG after Treatment with Various Concentrations of TAU (a) or TMZ (b) or the combinations (c) for 24 and $48 \mathrm{~h}$, after that, the $\mathrm{IC}_{50}$ value of TAU or TMZ was calculated. The histogram is presented in mean \pm SEM. $\$ p<0.05, \$ \$ p<0.01$ compared with the group treated only by TAU 24 mM (TAU24) at same time.
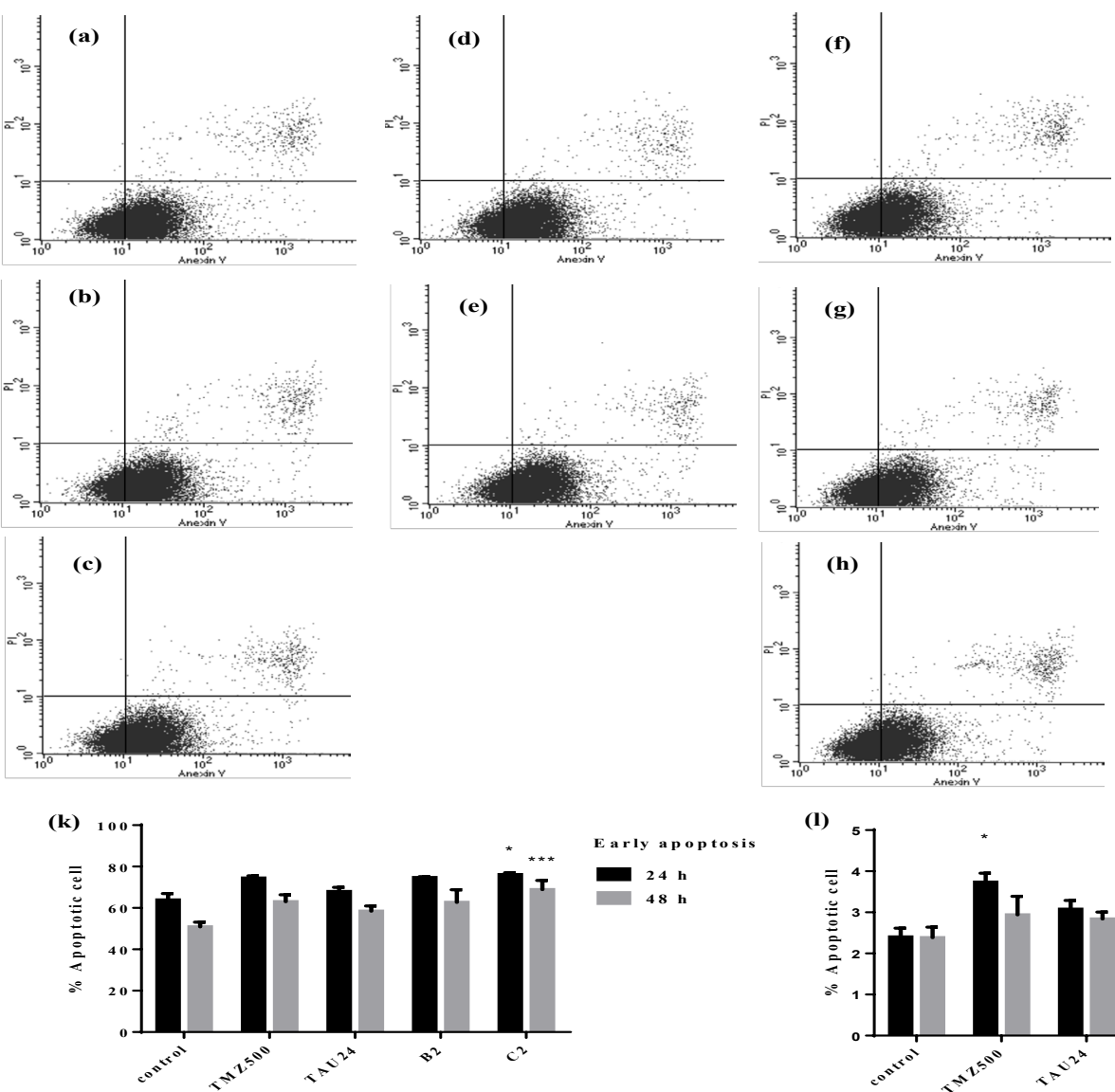

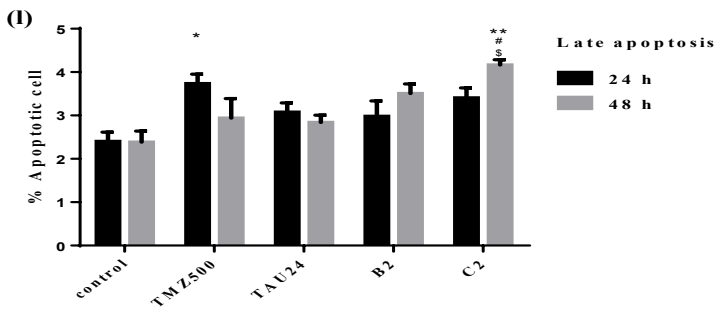

Figure 2. The Apoptotic Effects of the Combinations in U-251 MG Cells were Observed by Using Flow Cytometry. (aj) represent the results of cells incubated with 0 , TMZ $500 \mu \mathrm{M}$, TAU $24 \mathrm{mM}, \mathrm{B} 2$, and C2 for $24 \mathrm{~h}$ (a-e) and $48 \mathrm{~h}$ (f-j), respectively. The numbers of AnnexinV-FITC and PI-positive cells are shown in the scattered plot. The percentage of cell populations in early (k) and late (1) apoptotic stages per total cells at $24 \mathrm{~h}$ and $48 \mathrm{~h}$ are shown in the histograms. $* p<0.05, * * p<0.01, * * * p<0.001$ compared with control at same time. $\# p<0.05$ compared with TMZ500 at same time. $\$ \mathrm{p}<0.05$ compared with TAU24 at same time. 
(a)

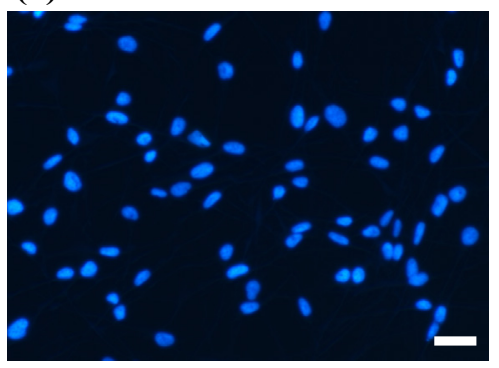

(b)

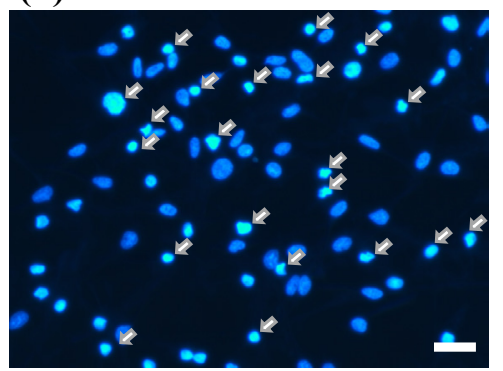

(c)

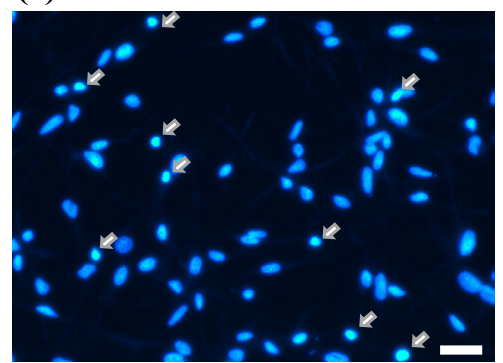

(d)

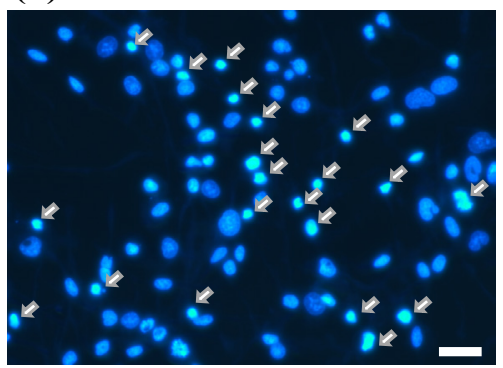

(e)

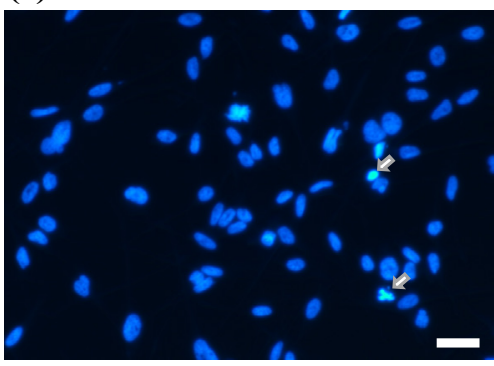

(f)

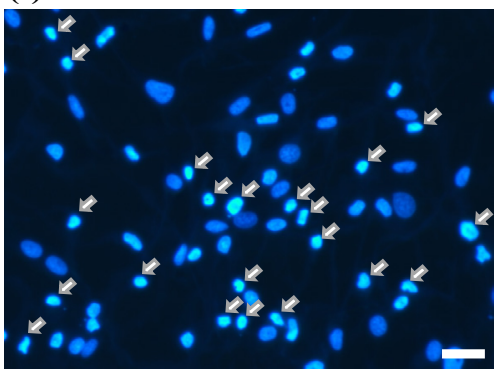

(g)

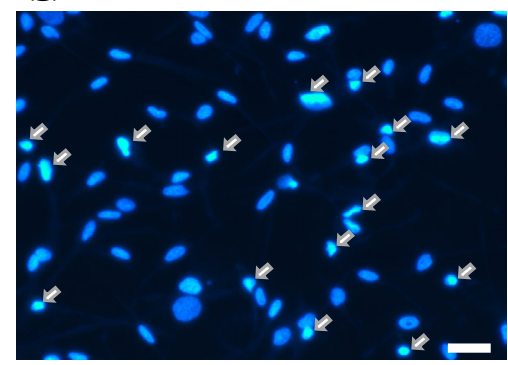

(h)

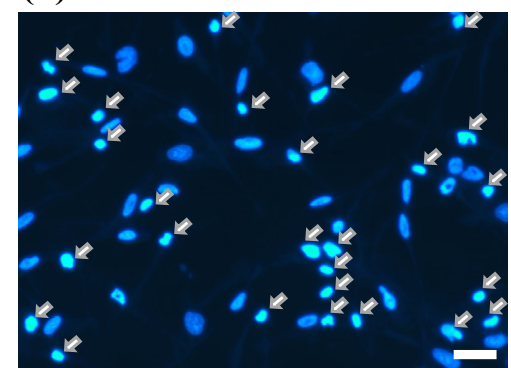

Figure 3. Detection of Condensed Chromosomes and Fragmented Nuclei in Treated U-251 MG Cells. (a-h) represents the results of cells stained with Hoechst 33342 and further visualized under a fluorescence microscope after incubated with 0, TAU $24 \mathrm{mM}$, TMZ $500 \mu \mathrm{M}$, and C2 for $24 \mathrm{~h}(\mathrm{a}-\mathrm{d})$ and $48 \mathrm{~h}(\mathrm{e}-\mathrm{h})$, respectively. Arrowheads display chromatin condensation and nuclear fragmentation. Scale bar $=50 \mu \mathrm{m}$.

either TAU or TMZ.

\section{Microscopic observation of U-251 MG cells stained with} Hoechst 33342

The staining with Hoechst 33342 was performed to examine the morphological changes in the nucleus of apoptotic cells. After treatment with TAU24, TMZ, and $\mathrm{C} 2$ for $24 \mathrm{~h}$ and $48 \mathrm{~h}$, the characteristics of apoptotic cells including condensed chromatin and fragmented nuclei were observed under a fluorescence microscope with bright bluish nuclei (Figure 3b-d, f-h), whereas the normal cells were illustrated with uniform staining (Figure 3a, e). As shown in Figure 3a-h, the result suggested that U-251 MG cells treated with $\mathrm{C} 2$ were enhanced in the number of apoptotic cells compared with those treated with TAU or TMZ alone.
Effect of the combinations on cell cycle distribution of U-251 MG cells

After U-251 MG cells were treated with the experimental substances, the cells were stained with PI and the distribution of each phase in the cell cycle was investigated using flow cytometry. The results revealed that the cells treated with TMZ, B2, and C2 could be induced a $\mathrm{G}_{2} / \mathrm{M}$ arrest only for $24 \mathrm{~h}$ treatment (Figure $4 \mathrm{~b}, \mathrm{~d}, \mathrm{e}, \mathrm{n})$. On the other hand, the percentage of cell distribution was significantly increased in the $\mathrm{SubG}_{1}$ phase when compared to a control group after $48 \mathrm{~h}$ treatment with TMZ, B2, and C2 (Figure 4k).

Expression analyses of apoptotic related proteins by western blot

Apoptotic effects at the protein expression level of the

Asian Pacific Journal of Cancer Prevention, Vol 22

4005 

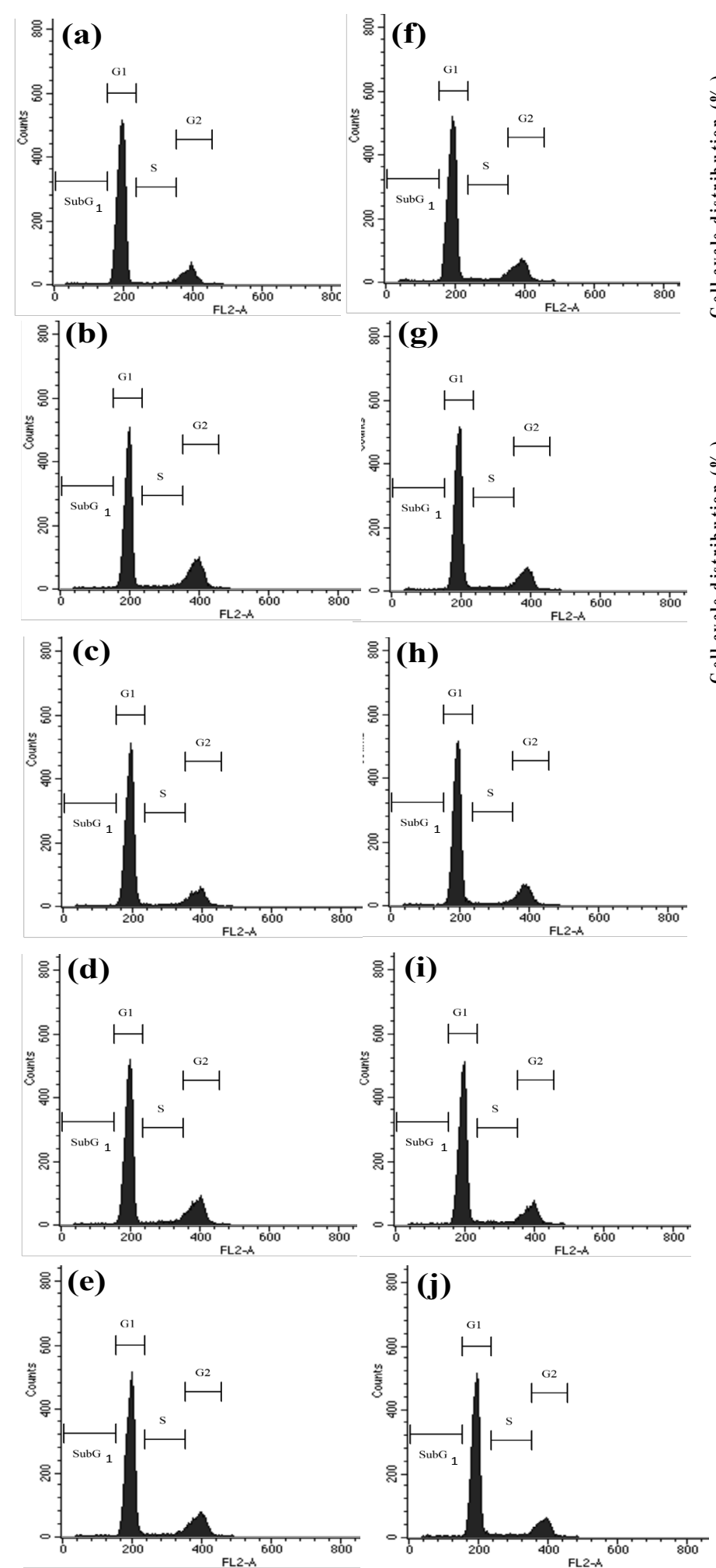

Figure 4. Cell Cycle Analyses of U-251 MG Cells Treated with a Single Agent, or the Combinations were Observed by Using Flow Cytometry. The distributions in various phases of the cell cycle of U-251 MG cells treated with 0 , TAU $24 \mathrm{mM}$, TMZ $500 \mu \mathrm{M}, \mathrm{B} 2$ and C2 for $24 \mathrm{~h}$ (a-e) and $48 \mathrm{~h}$ (f-j) are shown in the flow cytometric histograms, respectively. (k-n) represent the percentage of each phase as mean \pm SEM. ${ }^{*} \mathrm{p}<0.05, * * \mathrm{p}<0.01, * * * * \mathrm{p}<0.0001$ compared with control at same time. $\# \mathrm{p}<0.05$, \#\#p $<0.01$ compared with TMZ at same time. $\$ p<0.05, \$ \$ p<0.01$, $\$ \$ \$ p<0.0001$ compared with TAU at same time.

combinations in U-251 MG cells were also investigated. The expression levels of the apoptotic and anti-apoptotic markers were observed (Figure 5) after treatment with TAU, TMZ, B2, and C2 for $24 \mathrm{~h}$ and $48 \mathrm{~h}$. An apoptotic marker, BAX (Figure 5a, c) in cells treated with B2 and 4006 Asian Pacific Journal of Cancer Prevention, Vol 22
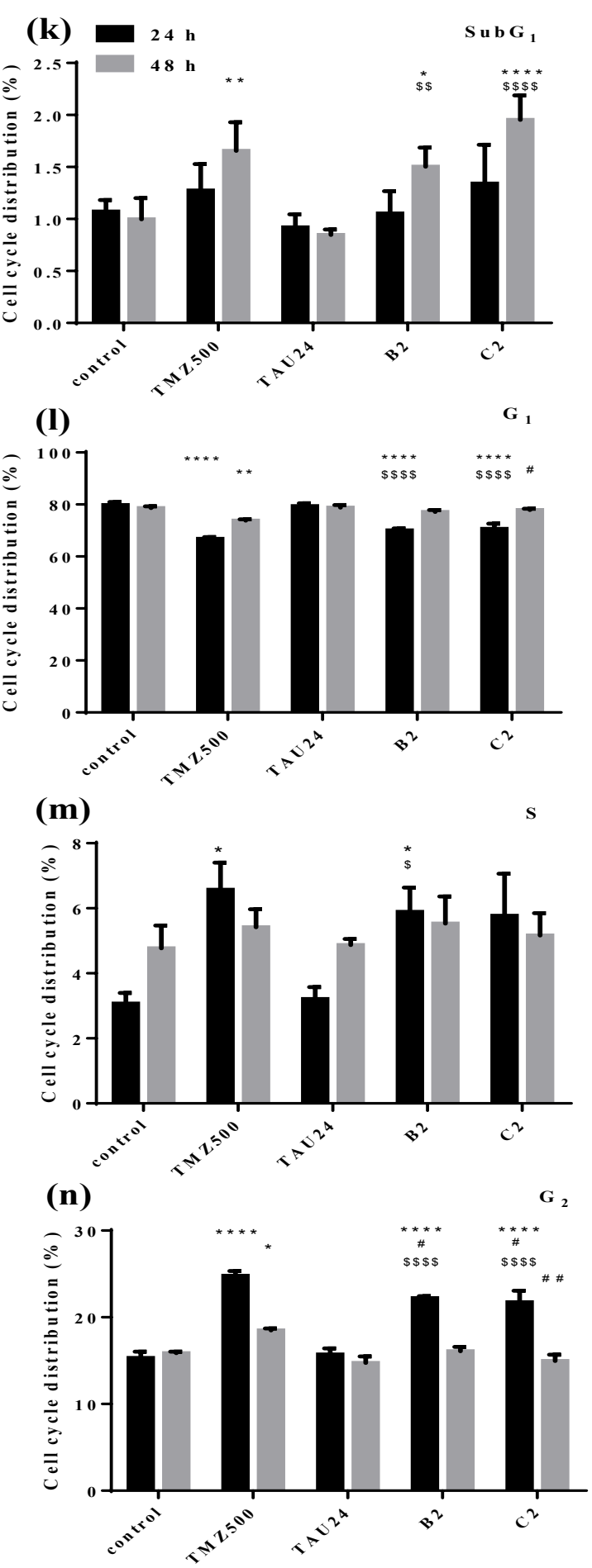

C2 were significantly increased at $48 \mathrm{~h}$ compared to a control group. In contrast, the expression of Bcl-2, an anti-apoptotic marker at $48 \mathrm{~h}$ was prone to decrease in C2 treatment (Figure 5a-b). Like Bcl-2, AKT expression was also prone to decrease after treatment for $48 \mathrm{~h}$ (Figure 
(a)

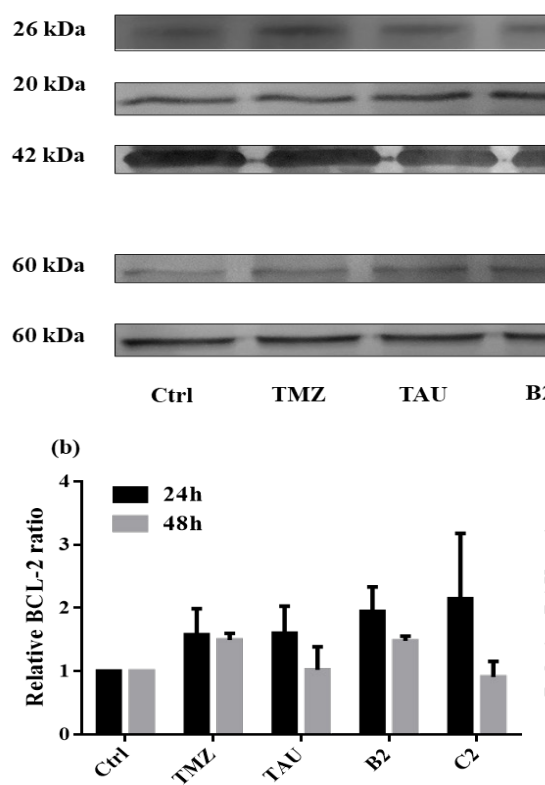

$24 \mathrm{~h}$

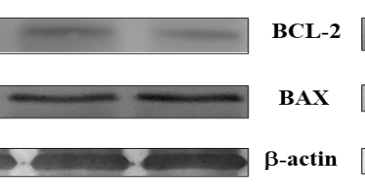

$48 \mathrm{~h}$

Figure 5. Expression of Apoptotic Related Proteins by Western Blotting Analysis in U-251 MG Cells Treated with 0, TAU $24 \mathrm{mM}$, TMZ $500 \mu \mathrm{M}, \mathrm{B} 2$ and C2. (a) illustrates western blot analysis of Bax, Bcl-2, p-AKT and AKT protein expressions at $24 \mathrm{~h}$ and $48 \mathrm{~h}$. Relative expression levels of (b) Bcl-2, (c) Bax, (d) the ratio of p-AKT, and AKT were analyzed and compared with the control group at the same time. $* \mathrm{p}<0.05$

5a, d). However, the expressions of Bcl-2 and AKT were non-significantly decreased compared to a control group.

\section{Discussion}

GBM is one of tumor that is difficult to treat because of its high resistance to TMZ which is the standard regimen for GBM treatment (Bleeker et al., 2012). Thus, the combination of therapeutic agents is a path to enhance therapeutic efficacy in this tumor. However, no study applies the combination between TAU and TMZ in GBM therapy. Therefore, the aim of this study was to examine the antitumor effects of TMZ and TAU combination on GBM.

The inhibition of cell proliferation was indicated as cytotoxicity of the substances. Previous studies revealed that TAU inhibited cell proliferation in various cancer cells, for example, colon cancer cells (Zhang et al., 2014; Liu et al., 2018), breast cancer cells (Zhang et al., 2015), lung cancer cells (Tu et al., 2018), nasopharyngeal carcinoma cells (He et al., 2018), Ehrlich ascites carcinoma (Ibrahim et al., 2018). In the present study, we found that TAU also inhibited cell proliferation in U-251 MG cells in a dose-dependent manner. Despite, TMZ is a major drug in the treatment of GBM, but the resistant rate of TMZ has highly occurred. Therefore, TMZ in several studies had been applied to combine with other agents including resveratrol (Lin et al., 2012), chloroquine (Lee et al, 2015), curcumin (Zanotto-Filho et al., 2015), rutin (Zhang et al., 2017), and caffeine (Li et al., 2018) as new strategies in GBM treatment. The present results revealed that TMZ could not inhibit cell proliferation at a high concentration of $500 \mu \mathrm{M}$. This referred that $\mathrm{U}-251$ MG might be resistant to TMZ. However, the combination between TMZ and TAU showed a synergistic effect on inhibition of tumor cell viability. By Chou-Talalay method to evaluate the interactions, ranges of $\mathrm{CI}$ including 0.85 $0.90,0.7-0.85,0.7-0.3,0.1-0.3$, and $<0.1$ can represent the degree of synergism comprising of slight synergism, moderate synergism, synergism, strong synergism, and very strong synergism, respectively (Chou and Martin, 2005). The combinations C2 (TAU (12mM) and TMZ $(375 \mu \mathrm{M})$ ratio TAU:TMZ $=32: 1)$ showed the strongest synergism $(\mathrm{CI}=0.69177)$ among others at $48 \mathrm{~h}$ after treatment. Moreover, results showed that $\mathrm{C} 2$ significantly decreased cell viability when compared to control or TMZ $(500 \mu \mathrm{M})$ alone and cytotoxicity of C2 was comparable with TAU $(24 \mathrm{mM})$ alone. According to the results, the dosages of combined agents were reduced as well as provided cytotoxicity effect on U-251 MG cells similar to single-agent with the higher dose. Therefore, the dose reduction with equivalent therapeutic effect might be a way to improve the strategy of treatment by adverse drug reaction reduction of chemotherapeutic drugs.

In the present study, it was revealed that the combination between TMZ and TAU induced U-251 MG cell apoptosis by the increase of nuclear chromatin condensation, DNA fragmentation, and Annexin V and PI-positive cells. Apoptosis is well known as programmed cell death. Basically, the molecules in cells involving apoptotic balance include Bcl-2, an anti-apoptotic protein, and Bax proteins, pro-apoptotic protein (Adams and Cory, 2007). Apart from Bcl-2 and Bax, Akt also regulates apoptosis at a postmitochondrial level by inhibiting activation of caspase- 9 and -3 which are the effector molecules for the apoptotic process (Zhou et al., 2000). The present study found that $\mathrm{C} 2$ has a synergistic effect to significantly induce apoptosis in U-251 MG cells at $48 \mathrm{~h}$ after treatment. 
However, we also found that Bcl-2 and Akt proteins were non-significantly down-regulated in U-251 MG cells. In contrast, Bax protein was significantly upregulated after treatment with B2 and C2 for $48 \mathrm{~h}$. Thus, the results suggested that apoptosis by the combination of TMZ and TAU might not relate with downregulation of Bcl-2 and Akt proteins in U-251 MG cells. Apart from Bcl-2, previous studies reported that PUMA (p53-upregulated modulator of apoptosis), a member of the BH3-only subgroup of Bcl-2 family proteins and a potent proapoptotic protein was upregulated after treatment with TAU in colorectal cancer cells (Zhang et al., 2014), breast cancer cell (Zhang et al., 2015), and lung cancer A549 cell (Tu et al., 2018) to induce apoptosis. Thus, PUMA should be further investigated for other mechanisms of apoptosis.

A characteristic of cancer cells is the high proliferative rate associated with some mutations, especially at regulatory genes of the cell cycle resulting in cell avoiding from the checkpoint mechanisms and then occurring the unlimited proliferation of cancer cells (DiPaola et al., 2002). In the present study using flow cytometry, we found that $\mathrm{a}_{2} / \mathrm{M}$ arrest in the cell cycle was induced after treatment with TMZ, B2, or C2 for $24 \mathrm{~h}$. Then, contents of DNA fragments were significantly increased in the subG $_{1}$ phase after treatment with TMZ, B2, or C2 for 48 h. These results suggested that $\mathrm{G}_{2} / \mathrm{M}$ arrest might induce apoptotic cells indicated by DNA fragment content in the subG $_{1}$ phase after treatment with $\mathrm{C} 2$ for $48 \mathrm{~h}$. Increasing apoptotic cells after treatment with $\mathrm{C} 2$ for $48 \mathrm{~h}$ in cell cycle investigation had the same trend as Annexin $\mathrm{V}$ and PI-positive cells. In other studies, they have previously been reported that the treatment of GBM cells with TMZ or agents combined with TMZ induced cell cycle arrest at $\mathrm{G}_{2} / \mathrm{M}$ phase (Li et al., 2018; Lee et al., 2015) and increased apoptotic cells in a time-dependent manner (Zhang et al., 2012; Zanotto-Filho et al., 2015).

In conclusion, all results presented in this study reveal that the combination between TMZ and TAU provides a synergistic effect to enhance cytotoxicity on U-251 MG cells in term of cell proliferation inhibition and apoptosis induction. These results provide a novel strategy for improving the antitumor activity of TMZ and TAU against GBM in the future.

\section{Author Contribution Statement}

Study conception and design: Thanakorn Surarak. Data collection: Thanakorn Surarak. Analysis and interpretation of the results: Thanakorn Surarak. Draft Manuscript Preparation: Thanakorn Surarak, Pathanin Chantree, and Kant Sangpairoj. All authors reviewed the results and approved the final version of the manuscript.

\section{Acknowledgements}

\section{Funding statement}

This study was supported by a research fund from the Faculty of Medicine, Thammasat University, Thailand (Contract No. 2-09/2562) to Thanakorn Surarak and a research grant of Thammasat University Research Unit in Nutraceuticals and Food Safety, Faculty of Medicine,
Thammasat University, Pathumthani, Thailand.

Data Availability

All relevant data are within the paper.

Declaration of Conflicts of Interest

The authors declare no conflict of interest.

\section{References}

Adams JM, Cory S (2007). Bcl-2-regulated apoptosis: mechanism and therapeutic potential. Curr Opin Immunol, 19, 488-96.

Bleeker FE, Molenaar RJ, Leenstra S (2012). Recent advances in the molecular understanding of glioblastoma. J Neurooncol, 108, 11-27.

Chou TC (2010). Drug combination studies and their synergy quantification using the Chou-Talalay method. Cancer Res, 70, $440-6$.

Chou TC, Martin N (2005). Scope of applications. In 'CompuSyn for drug combination and for general dose-effect analysis: user's guide', Eds 34.

Curran CP, Marczinski CA (2017). Taurine, caffeine, and energy drinks: Reviewing the risks to the adolescent brain. Birth Defects Res, 109, 1640-8.

DiPaola RS (2002). To arrest or not to G(2)-M Cell-cycle arrest : commentary re: A. K. Tyagi et al., Silibinin strongly synergizes human prostate carcinoma DU145 cells to doxorubicin-induced growth inhibition, $\mathrm{G}(2)-\mathrm{M}$ arrest, and apoptosis. Clin Cancer Res, 8, 3512-9, 2002.

Gao P, Yang C, Nesvick CL, et al (2016). Hypotaurine evokes a malignant phenotype in glioma through aberrant hypoxic signaling. Oncotarget, 7, 15200-14.

Goron A, Moinard C (2018). Amino acids and sport: a true love story?. Amino Acids, 50, 969-80.

He F, Ma N, Midorikawa K, et al (2018). Taurine exhibits an apoptosis-inducing effect on human nasopharyngeal carcinoma cells through PTEN/Akt pathways in vitro. Amino Acids, 50, 1749-58.

Ibrahim HM, Abdel Ghaffar FR, El-Elaimy IA, et al (2018). Antitumor and immune-modulatory efficacy of dual-treatment based on levamisole and/or taurine in Ehrlich ascites carcinoma-bearing mice. Biomed Pharmacother, 106, 43-9.

Lee SW, Kim HK, Lee NH, et al (2015). The synergistic effect of combination temozolomide and chloroquine treatment is dependent on autophagy formation and $\mathrm{p} 53$ status in glioma cells. Cancer Lett, 360, 195-204.

Lee SY (2016). Temozolomide resistance in glioblastoma multiforme. Genes Dis, 3, 198-210.

Li N, Zhang P, Kiang KMY, et al (2018). Caffeine sensitizes U87-MG human glioblastoma cells to temozolomide through mitotic catastrophe by impeding G2 arrest. Biomed Res Int, 2018, 5364973.

Lin CJ, Lee CC, Shih YL, et al (2012). Resveratrol enhances the therapeutic effect of temozolomide against malignant glioma in vitro and in vivo by inhibiting autophagy. Free Radic Biol Med, 52, 377-91.

Liu Z, Xia Y, Zhang X, et al (2018). Roles of the MST1-JNK signaling pathway in apoptosis of colorectal cancer cells induced by Taurine. Libyan J Med, 13, 1500346.

Miranda-Filho A, Pineros M, Soerjomataram I, et al (2017). Cancers of the brain and CNS: global patterns and trends in incidence. Neuro Oncol, 19, 270-80.

Schaffer S, Kim HW (2018). Effects and mechanisms of taurine as a therapeutic agent. Biomol Ther (Seoul), 26, 225-41. 
Seidel U, Huebbe P, Rimbach G (2019). Taurine: A regulator of cellular redox homeostasis and skeletal muscle function. Mol Nutr Food Res, 63, e1800569.

Shergalis A, Bankhead A, 3rd, Luesakul U, et al (2018). Current challenges and opportunities in treating glioblastoma. Pharmacol Rev, 70, 412-45.

Tu S, Zhang XL, Wan HF, et al (2018). Effect of taurine on cell proliferation and apoptosis human lung cancer A549 cells. Oncol Lett, 15, 5473-80.

Urbanska K, Sokolowska J, Szmidt M, et al (2014). Glioblastoma multiforme - an overview. Contemp Oncol (Pozn), 18, 307-12.

Witthayanuwat S, Pesee M, Supaadirek C, et al (2018). Survival analysis of glioblastoma multiforme. Asian Pac J Cancer Prev, 19, 2613-7.

Zanotto-Filho A, Braganhol E, Klafke K, et al (2015). Autophagy inhibition improves the efficacy of curcumin/temozolomide combination therapy in glioblastomas. Cancer Lett, 358, 220-31.

Zhang J, Stevens MF, Bradshaw TD (2012). Temozolomide: mechanisms of action, repair and resistance. Curr Mol Pharmacol, 5, 102-14.

Zhang P, Sun S, Li N, et al (2017). Rutin increases the cytotoxicity of temozolomide in glioblastoma via autophagy inhibition. J Neurooncol, 132, 393-400.

Zhang X, Lu H, Wang Y, et al (2015). Taurine induces the apoptosis of breast cancer cells by regulating apoptosisrelated proteins of mitochondria. Int J Mol Med, 35, 218-26.

Zhang X, Tu S, Wang Y, et al (2014). Mechanism of taurineinduced apoptosis in human colon cancer cells. Acta Biochim Biophys Sin (Shanghai), 46, 261-72.

Zhou H, Li XM, Meinkoth J, et al (2000). Akt regulates cell survival and apoptosis at a postmitochondrial level. $J$ Cell Biol, 151, 483-94.

\section{(c) (7) (8)}

This work is licensed under a Creative Commons AttributionNon Commercial 4.0 International License. 